\title{
The prognostic value of ADRA1 subfamily genes in gastric carcinoma
}

\author{
TINGAN WANG ${ }^{*}$, YUZHOU QIN*, HAO LAI, WEIYUAN WEI, ZHAO LI, YANG YANG, \\ MINGWEI HUANG and JIANSI CHEN
}

Department of Gastrointestinal Surgery, Affiliated Tumour Hospital of Guangxi Medical University, Nanning, Guangxi 530021, P.R. China

Received September 19, 2018; Accepted June 4, 2019

DOI: $10.3892 / 01.2019 .10660$

\begin{abstract}
Adrenergic receptor $\alpha 1$ (ADRA1) subfamily members, including ADRA1A, ADRA1B and ADRA1D, are understood to participate in cardiac disease and benign prostatic hyperplasia. In addition, adrenergic signals in cell pathways can promote the development of cancer. However, little is understood regarding the associations between ADRA1 subfamily members and gastric carcinoma (GC). The present study investigated the prognostic value of the ADRA1 subfamily genes in GC. Data from a total of 379 patients with GC were obtained from The Cancer Genome Atlas and Gene Expression Omnibus databases. Kaplan-Meier analysis and Cox regression analysis were used to determine associations with overall survival (OS) and to evaluate the median survival time using hazard ratios (HRs) and 95\% confidence intervals (CIs). Multivariate survival analysis revealed that low expression levels of ADRA1A (HR, 0.595; 95\% CI, 0.426-0.831; adjusted $\mathrm{P}=0.002)$ ADRA1B (HR, 0.576; 95\% CI, 0.412-0.805; adjusted $\mathrm{P}=0.001)$ and $\mathrm{ADRA} 1 \mathrm{D}(\mathrm{HR}, 0.559 ; 95 \% \mathrm{CI}$, $0.398-0.787$; adjusted $\mathrm{P}=0.001$ ) were associated with a favourable OS. Joint-effects analysis demonstrated that combinations of low expression levels of ARDA1A, ARDA1B and ARDA1D were significantly associated with a favourable OS. Overall, the current results suggested that the mRNA expression levels of ARDA1 subfamily members may serve as potential prognostic markers for GC.
\end{abstract}

Correspondence to: Professor Jiansi Chen or Dr Mingwei Huang, Department of Gastrointestinal Surgery, Affiliated Tumour Hospital of Guangxi Medical University, 6 Shuangyong Road, Nanning, Guangxi 530021, P.R. China

E-mail: jiansichen@126.com

E-mail: mingweihuang1@163.com

${ }^{*}$ Contributed equally

Key words: adrenergic receptor $\alpha 1$ subfamily, mRNA expression, gastric carcinoma, prognosis

\section{Introduction}

Gastric carcinoma (GC) is one of the most common types of malignancy and is the second leading cause of cancer-related mortality worldwide (1). Despite advances in surgical techniques, adjuvant chemotherapy, radiotherapy and immune therapy, the morbidity and mortality rates remain high (2). Therefore, new strategies to improve the diagnosis and prognosis of GC are urgently required.

Tumour metastasis is a complex process that serves a vital role in the progression and outcome of the cancer (3). Therefore, novel effective strategies that target metastasis are required. Previous studies have suggested that adrenergic receptor antagonists could be used in novel therapeutic approaches in the treatment of various types of cancer, including prostate and breast carcinoma $(4,5)$. Tumour metastasis involves the migration of cancer cells from the primary tumour via lymphoid/hematopoietic pathways and is regulated by exogenous signalling molecules, including $G$ protein coupled receptor (GPCR) ligands, chemokines and neurotransmitters $(6,7)$. A previous study has reported that norepinephrine, a stress-associated neurotransmitter, is a potent inducer of migration in cancer cell lines (8).

Adrenergic receptor antagonists include two major groups; $\alpha$ receptor and $\beta$ receptor specific antagonists $(9,10)$. Adrenergic receptor $\alpha 1$ (ADRA1) receptor is classified into three different subtypes ADRA1A, ADRA1B and ADRA1D, that differ in their tissue distribution, cell signalling, pharmacology and physiological roles (11). ADRA1 receptors are ubiquitous in the majority of human tissues; ADRA1B is predominantly expressed on the cell surface, ADRA1A is mainly expressed on the cell surface and intracellularly, and ADRA1D is primarily localized perinuclear (12).

It is well-known that ADRA1 is a member of the GPCR family, which interacts with a heterotrimeric $G$ protein containing the Gaq/11/14/16 subunits (13). The Gaq subunit is the main activator of phospholipase $\mathrm{CE}$, which promotes the cleavage of phosphatidylinositol-4,5-diphosphate into diacylglycerol and inositol-1,4,5-triphosphate. This subsequently promotes the release of $\mathrm{Ca}^{2+}$ from intracellular stores to activate protein kinase C (PKC) (14).

Previous studies have suggested that adrenergic signals in cells can promote the development of cancer $(15,16)$. However, 
little is understood regarding the associations between the expression levels of ARDA1A, ARDA1B and ARDA1D and the risk of GC. Therefore, the aim of the present study was to identify the associations between the expression levels of individual ADRA1 subtypes and GC prognosis. The current study may provide insights into the potential functional roles of ADRA1 subtypes in GC.

\section{Materials and methods}

Patient and disease characteristics. Metabolic gEne Rapid Visualizer (http://merav.wi.mit.edu/) was used to generate boxplots of the expression levels of ADRA1 subtypes in normal gastric tissues and primary GC tumours. Subsequently, clinical data were obtained for 379 patients with GC, including sex, grade (17), age, Tumour-Node-Metastasis (TNM) stage (18), targeted therapy, events (metastases or deaths), survival time, mortality status and mRNA expression levels of ADRA1A, ADRA1B and ADRA1D, from OncoLnc (http://www.oncolnc. org/) and Gene Expression Omnibus (GEO; https://www. ncbi.nlm.nih.gov/geo/query/acc.cgi?acc=GSE14520) databases $(19,20)$. The data presented here are based on studies published on The Cancer Genome Atlas (TCGA) $(21,22)$. To analyse the difference in the expression levels in cancer compared with the adjacent tissues, three genes (ADRA1A, ADRA1B and ADRA1D0 were searched in the GEPIA (http://gepia.cancer-pku.cn/) database separately.

Correlation and functional enrichment analysis of the ADRAI subfamily. The online Database for Annotation, Visualization, and Integrated Discovery (DAVID; v.6.8; https://david.ncifcrf. gov/tools.jsp) (23) was used to perform functional enrichment. This included gene ontology (GO) functional analysis and Kyoto Encyclopaedia of Genes and Genomes (KEGG) pathway analysis. In the present study, GO functional analysis consisted of molecular function, cellular component, and biological process terms. KEGG pathway analysis was performed on the ADRA1 gene subfamily. The gene function prediction website GeneMANIA (http://genemania.org/) was used to construct gene-gene interaction networks (24).

Survival analysis. Using the TCGA data, 379 patients with GC were divided into high- and low-expression groups according to the $50 \%$ cut-off values (median values). Overall survival (OS) and median survival time (MST) were applied to estimate patient prognosis. Kaplan-Meier analysis with a log-rank test was used to identify associations between ADRA1A, ADRA1B and ADRA1D mRNA expression levels and patient survival. Cox regression analysis was then used to evaluate statistically significant factors, including age and TNM stage.

Joint-effects analysis. In the TCGA database, the expression levels of ADRA1A, ADRA1B and ADRA1D were significantly different between tumour and non-tumour tissues. Therefore, joint-effects analysis was performed with the following combinations: i) ADRA1A and ADRA1B; i) ADRA1A and ADRA1D; iii) ADRA1B and ADRA1D; and iv) ADRA1A, ADRA1B and ADRA1D (Table I). A Cox regression model was adjusted for TNM stage, age and sex in keeping with the aforementioned combinations.
Table I. Grouping according to the expression levels of two or three selected genes.

\begin{tabular}{ll}
\hline Group & \multicolumn{1}{c}{ Composition } \\
\hline I & Low ARDA1A + low ARDA1B \\
II & Low ARDA1A + high ARDA1B \\
& High ARDA1A + low ARDA1B \\
III & High ARDA1A + high ARDA1B \\
IV & Low ARDA1A + low ARDA1D \\
V & Low ARDA1A + high ARDA1D \\
& High ARDA1A + low ARDA1D \\
VI & High ARDA1A + high ARDA1D \\
VII & Low ARDA1B + low ARDA1D \\
VIII & Low ARDA1B + high ARDA1D \\
& High ARDA1B + low ARDA1D \\
IX & High ARDA1B + high ARDA1D \\
X & Low ARDA1A + low ARDA1B + low ARDA1D \\
XI & High ARDA1A + low ARDA1B + high ARDA1D \\
& Low ARDA1A + high ARDA1B + high ARDA1D \\
& Low ARDA1A + low ARDA1B + low ARDA1D \\
& High ARDA1A + high ARDA1B + high ARDA1D \\
& High ARDA1A + low ARDA1B + low ARDA1D \\
& Low ARDA1A + high ARDA1B + low ARDA1D \\
XII & High ARDA1A + high ARDA1B + high ARDA1D \\
\end{tabular}

ADRA1, adrenergic receptor $\alpha 1$.

Statistical analysis. OS was evaluated by Kaplan-Meier survival analysis followed by a log-rank test. Cox proportional hazards regression model was used for univariate and multivariate survival analysis. GraphPad Prism v.7.0 (GraphPad Prism, Inc.) was used to construct vertical scatter plots and survival curves. All statistical analyses were performed using SPSS v.22.0 software and compared using an independent-samples t-test (IBM, Inc.). $\mathrm{P}<0.05$ was considered to indicate a statistically significant difference.

\section{Results}

Clinicopathological features of the patient cohort. Detailed characteristics of the 379 included patients obtained from the TCGA database are presented in Table II. It was identified that TNM stage and age were significantly associated with MST $(\mathrm{P}<0.001$ and $\mathrm{P}=0.007$, respectively); however, no significant associations were revealed for grade, sex or targeted therapy (all P>0.05; Table II).

Analysis of ADRAl subfamily gene expression in GC. Expression levels of ADRA1A, ADRA1B and ADRA1D in primary GC tissues and normal tissues were analysed using the online tool (GEPIA; http:/gepia.cancer-pku.cn/) and illustrated by boxplots (Fig. 1A-C). The expression levels of ADRA1A were higher in normal gastric tissues compared with primary gastric tumours. However, no marked differences in the expression levels of ADRA1B and ADRA1D were revealed between primary GC tumours and normal 
Table II. Clinical data for the 379 patients with gastric carcinoma.

\begin{tabular}{|c|c|c|c|c|c|}
\hline Variable & Patients $(n=379)$ & No. of events (\%) & MST, days & $\mathrm{HR}(95 \% \mathrm{CI})$ & Log-rank P-value \\
\hline \multicolumn{6}{|l|}{ Grade } \\
\hline $1+2$ & 146 & $52(35.6)$ & 1,294 & Ref. & \multirow[t]{2}{*}{0.07} \\
\hline 3 & 233 & $99(42.5)$ & 801 & $1.333(0.977-1.818)$ & \\
\hline \multicolumn{6}{|l|}{ Sex } \\
\hline Male & 247 & $104(42.1)$ & 2,030 & Ref. & \multirow[t]{2}{*}{0.229} \\
\hline Female & 132 & 47 (35.6) & 874 & $1.236(0.875-1.745)$ & \\
\hline \multicolumn{6}{|c|}{ Age, years } \\
\hline$\geq 60$ & 124 & $38(30.6)$ & 475 & Ref. & \multirow[t]{2}{*}{0.007} \\
\hline$<60$ & 255 & $113(44.3)$ & 792 & $0.604(0.418-0.874)$ & \\
\hline \multicolumn{6}{|c|}{ TNM stage } \\
\hline I & 50 & $12(24.0)$ & 2,197 & $0.270(0.133-0.550)$ & \multirow[t]{5}{*}{$<0.001$} \\
\hline II & 119 & $33(27.7)$ & 1,686 & $0.372(0.214-0.645)$ & \\
\hline III & 164 & $78(47.6)$ & 766 & $0.649(0.400-1.053)$ & \\
\hline IV & 34 & $21(61.8)$ & 476 & Ref. & \\
\hline NA & 12 & & & & \\
\hline \multicolumn{6}{|c|}{ Targeted therapy } \\
\hline No & 188 & $79(42.0)$ & 805 & Ref. & \multirow[t]{3}{*}{0.063} \\
\hline Yes & 162 & $59(38.4)$ & 1,294 & $0.726(0.518-1.018)$ & \\
\hline NA & 29 & & & & \\
\hline
\end{tabular}

MST, median survival time; HR, hazard ratio; CI, confidence interval; Ref, reference group; NA, not available; TNM, Tumor-Node-Metastasis.
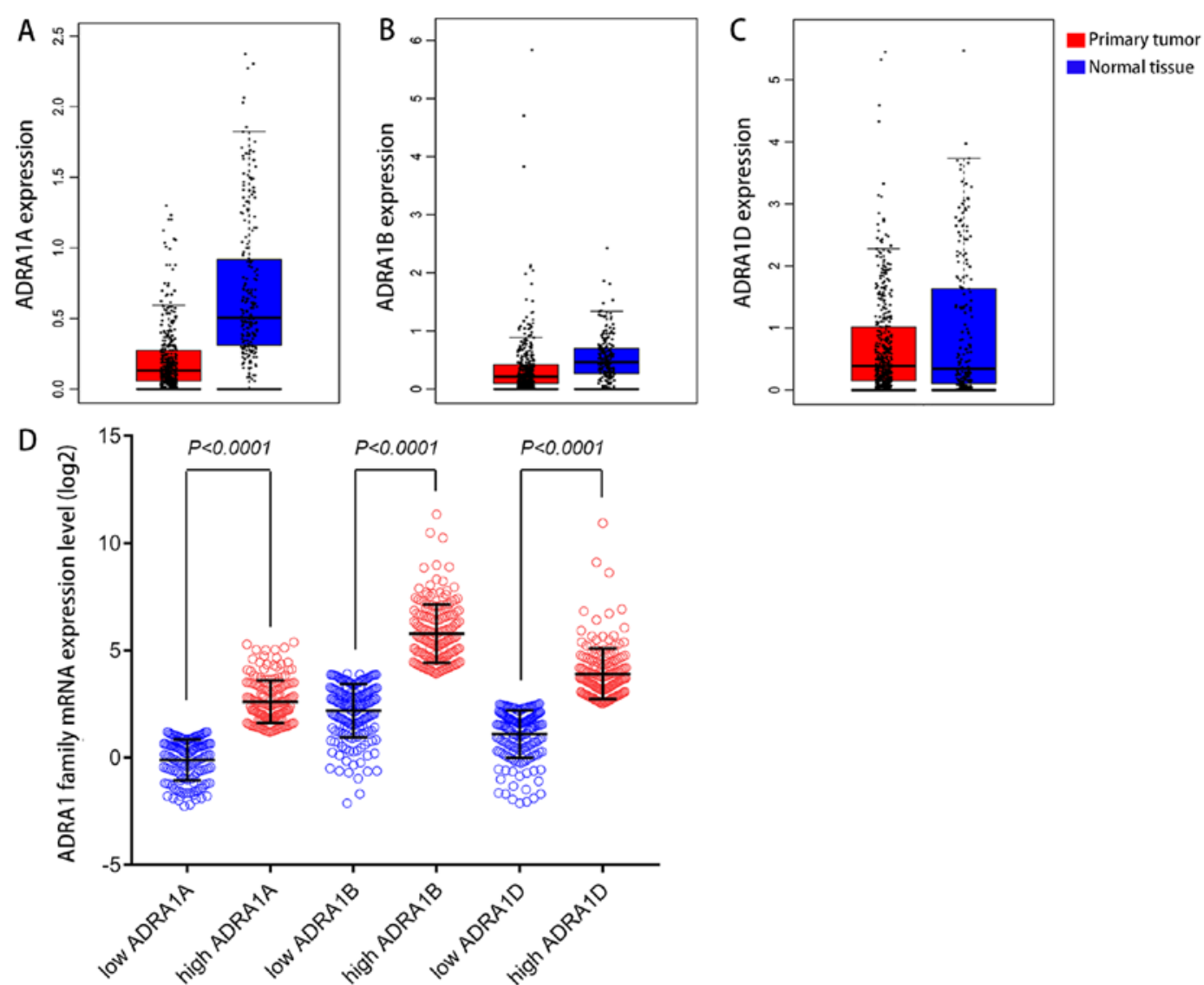

Figure 1. Boxplots for ADRA1 gene expression in normal gastric tissue and primary gastric cancer tissue. (A) ADRA1A expression. (B) ADRA1B expression. (C) ADRA1D expression. (D) Scatter plots for ADRA1A, ADRA1B and ADRA1D gene expression levels from data obtained from The Cancer Genome Atlas. ADRA1, adrenergic receptor $\alpha 1$. 

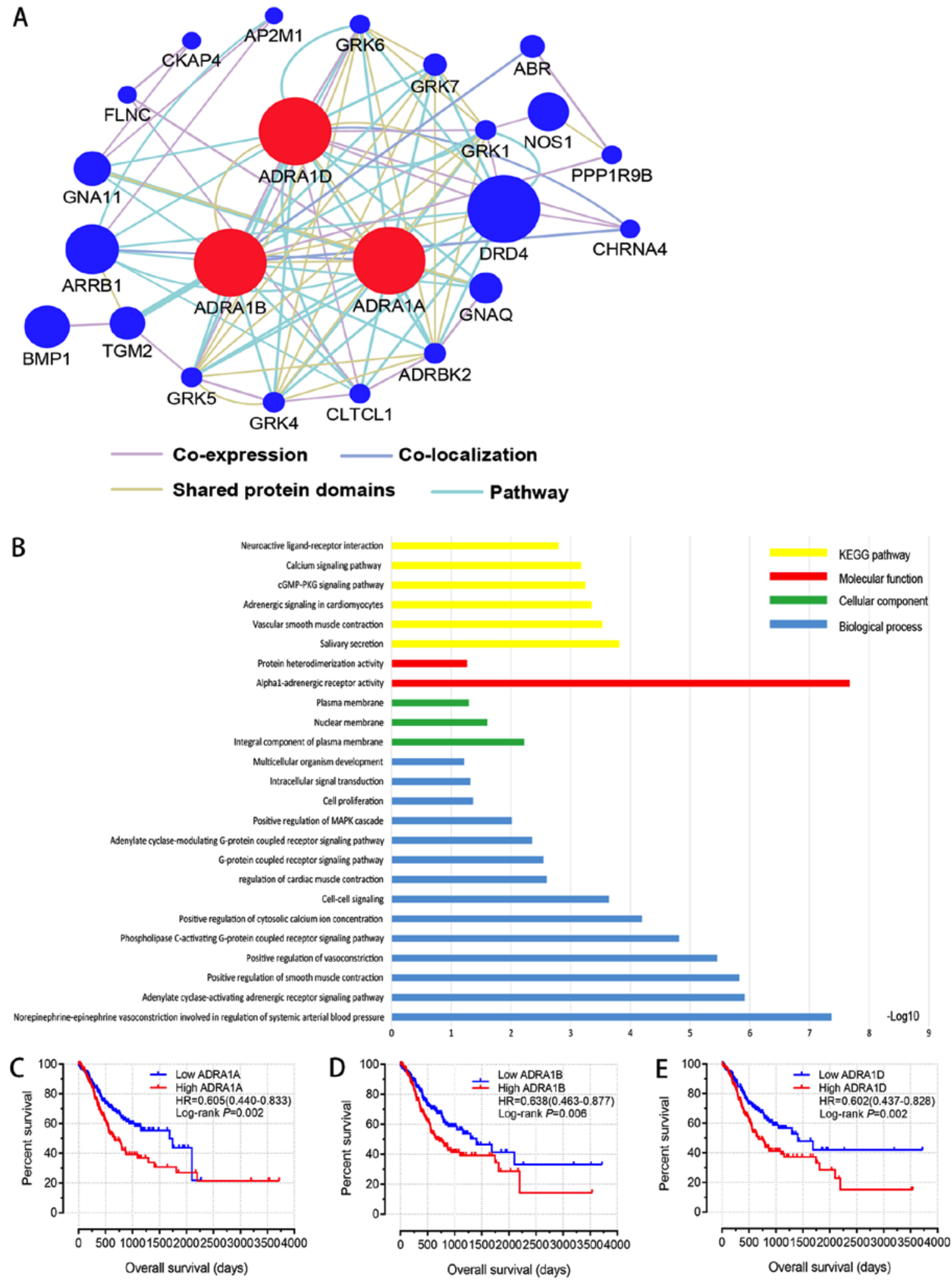

Figure 2. Prognostic value, interaction network and the function of the ADRA1 subfamily of genes. (A) Protein-interaction network among selected genes generated by GeneMANIA. (B) Analysis of enriched gene ontology terms and KEGG pathways for ADRA1 genes using Database for Annotation, Visualization, and Integrated Discovery. The prognostic value of (C) ADRA1A, (D) ADRA1B and (E) ADRA1D expression was analysed by Kaplan-Meier survival curves for 379 patients with gastric cancer. ADRA1, adrenergic receptor a1; KEGG, Kyoto Encyclopaedia of Genes and Genomes; HR, hazard ratio; cGMP-PKG, MAPK, mitogen-activated protein kinase.

tissues. The low expression groups and high expression groups for these genes obtained from the GEO and TCGA databases were analysed by Scatter diagrams and compared using an independent-samples t-test (Fig. 1D).
GO and KEGG pathway analysis of the ADRAl subfamily. The results of GO analysis of ADRA1A, ADRA1B and ADRA1D including molecular function, cellular component and biological process terms, are presented in Fig. 2B. KEGG 
Table III. Survival analysis according to the expression levels of ADRA1 subfamily genes.

\begin{tabular}{|c|c|c|c|c|c|c|c|}
\hline Gene & $\begin{array}{l}\text { Patients } \\
(\mathrm{n}=379)\end{array}$ & $\begin{array}{c}\text { No. of } \\
\text { events }(\%)\end{array}$ & $\begin{array}{l}\text { MST, } \\
\text { days }\end{array}$ & $\begin{array}{c}\text { Crude HR } \\
(95 \% \text { CI) }\end{array}$ & $\begin{array}{l}\text { Crude } \\
\text { P-value }\end{array}$ & $\begin{array}{l}\text { Adjusted HR } \\
(95 \% \mathrm{CI})^{\mathrm{a}}\end{array}$ & $\begin{array}{l}\text { Adjusted } \\
\text { P-value }^{\mathrm{a}}\end{array}$ \\
\hline \multicolumn{8}{|c|}{ ADRA1A } \\
\hline Low & 189 & $61(32.1)$ & 1,686 & Ref. & \multirow[t]{3}{*}{0.001} & Ref. & \multirow[t]{3}{*}{0.002} \\
\hline High & 189 & $90(47.6)$ & 669 & $0.601(0.434-0.834)$ & & $0.595(0.426-0.831)$ & \\
\hline NA & 1 & & & & & & \\
\hline \multicolumn{8}{|c|}{ ADRA1B } \\
\hline Low & 189 & $62(32.9)$ & 1,407 & Ref. & \multirow[t]{3}{*}{0.006} & Ref. & \multirow[t]{3}{*}{0.001} \\
\hline High & 189 & $89(47.1)$ & 712 & $0.637(0.460-0.881)$ & & $0.0576(0.412-0.805)$ & \\
\hline NA & 1 & & & & & & \\
\hline \multicolumn{8}{|c|}{ ADRA1D } \\
\hline Low & 189 & $58(30.7)$ & 1,407 & Ref. & \multirow[t]{3}{*}{0.002} & Ref. & \multirow[t]{3}{*}{0.001} \\
\hline High & 189 & $93(49.2)$ & 675 & $0.601(0.433-0.835)$ & & $0.559(0.398-0.787)$ & \\
\hline NA & 1 & & & & & & \\
\hline
\end{tabular}

${ }^{a}$ Adjusted for age and Tumour-Node-Metastasis stage. ADRA1, adrenergic receptor $\alpha 1$; MST, median survival time; HR, hazard ratio; CI, confidence interval; Ref, reference group; NA, not available.

enrichment analysis identified certain signalling pathways associated with ADRA1 subfamily members, such as 'Calcium signalling pathway', 'cGMP-PKG signalling pathway' and mitogen-activated protein kinase (MAPK) signalling (Fig. 2B). Furthermore, the GeneMANIA website (http://genemania. org/) was used to analyse interaction networks of ADRA1A, ADRA1B and ADRA1D. The interaction network between the ADRA1 subfamily and other genes presented in Fig. 2A.

Association of ADRA1 subfamily members with survival. Cox proportional hazards regression analysis of the ADRA1 members revealed that the mRNA expression levels of all three genes were significantly associated with MST in patients with GC (Table III). As aforementioned in Table I, TNM stage and age were also identified as prognostic factors in TCGA GC cohort. Therefore, a multivariate Cox regression model was employed next, in order to explore whether the expression levels of the ADRA1 subfamily genes were independent prognostic factors following adjustment for age and TNM stage. The results revealed that the lower expression levels of ADRA1A [hazard ratio (HR), 0.595; 95\% CI, 0.426-0.831; adjusted $\mathrm{P}=0.002$ ], ADRA1B (HR, 0.576; 95\% CI, 0.412-0.805; adjusted $\mathrm{P}=0.001$ ) and ADRA1D (HR, 0.559; 95\% CI, 0.398-0.787; adjusted $\mathrm{P}=0.001$ ) were significantly associated with favourable MST in patients with GC, following adjustment for age and TNM stage (Table III). The survival curves of ADRA1A, ADRA1B and ADRA1D are presented in Fig. 2C-E; these revealed that low expression levels of these genes were significantly associated with a favourable OS in patients with $\mathrm{GC}(\mathrm{P}=0.002, \mathrm{P}=0.006$ and $\mathrm{P}=0.002$, respectively).

Joint-effects analysis of the ADRA1 subfamily. Based on the influence of ADRA1A, ADRA1B and ADRA1D on patient survival identified by multivariate analysis, a joint-effects model was employed to evaluate the combined effects of these genes on the OS of patients with GC. According to the different expression levels of ADRA1A, ADRA1B and ADRA1D, 12 groups were generated, as presented in Table I. Group XII, with high expression levels of ARDA1A, ARDA1B and ARDA1D, exhibited the shortest MST of 552 days (adjusted $\mathrm{P}=0.001$ ). Group X, with low expression levels of ARDA1A, ARDA1B and ARDA1D, exhibited the highest MST of 2,100 days (adjusted $\mathrm{P}=0.002$ ). The detailed joint-effects analysis results are presented in Table IV.

Associated survival curves, presented in Fig. 3, were evaluated by Kaplan-Meier analysis with a log-rank test. Low expression levels of ADRA1A, ADRA1B and ADRA1D (group $\mathrm{X}$ ) were identified to be significantly associated with favourable OS. By contrast, high expression levels of ADRA1A, ADRA1B and ADRA1D (group XII) were revealed to be significantly associated with poor OS (all $\mathrm{P}<0.05$; Fig. 3 ).

\section{Discussion}

Cancer metastasis can be influenced by numerous factors, including intracellular signalling molecules and extracellular components, including cytokines, the extracellular matrix and neurotransmitters (25). It has previously been suggested that the nervous system serves an important role in the progression of cancer. It has been reported that the sympathetic and parasympathetic nervous systems participate in the development and distribution of cancer (26).

Adrenergic neurotransmitters, including norepinephrine (NE) and epinephrine (E), have been reported to promote cell migration and invasion in various types of cancer $(6,27)$. Previous studies have demonstrated that NE/E may promote tumour progression by promoting angiogenesis (27). Furthermore, the tumour stromal cells in the microenvironment of cancer may be affected by the nervous system (28). A number of neurotransmitter receptor-associated genes have been identified to be closely associated with the proliferation and survival of tumour cells by a large-scale complementary DNA transfection screening (29). 
Table IV. Joint-effects analysis of the prognostic value of combinations of ADRA1A, ADRA1B and ADRA1D expression levels in gastric carcinoma.

\begin{tabular}{lcrrcrc}
\hline Group & $\begin{array}{c}\text { Patients } \\
(\mathrm{n}=379)\end{array}$ & $\begin{array}{c}\text { MST, } \\
\text { days }\end{array}$ & $\begin{array}{c}\text { Crude } \\
\text { P-value }\end{array}$ & $\begin{array}{c}\text { Crude HR } \\
(95 \% \mathrm{CI})\end{array}$ & $\begin{array}{c}\text { Adjusted } \\
\text { P-value }^{\mathrm{a}}\end{array}$ & $\begin{array}{c}\text { Adjusted HR } \\
(95 \% \mathrm{CI})^{\mathrm{a}}\end{array}$ \\
\hline I & 148 & 1,407 & $<0.001$ & $0.480(0.327-0.705)$ & $<0.001$ & $0.444(0.297-0.664)$ \\
II & 114 & 2,100 & 0.005 & $0.552(0.364-0.838)$ & 0.004 & $0.531(0.347-0.813)$ \\
III & 113 & 560 & $<0.001$ & Ref. & $<0.001$ & Ref. \\
IV & 115 & 1,686 & $<0.001$ & $0.420(0.271-0.651)$ & $<0.001$ & $0.406(0.260-0.633)$ \\
V & 147 & 1,294 & 0.083 & $0.729(0.510-1.043)$ & 0.031 & $0.665(0.458-0.963)$ \\
VI & 114 & 633 & 0.001 & Ref. & $<0.001$ & Ref. \\
VII & 121 & 1,407 & $<0.001$ & $0.481(0.319-0.725)$ & $<0.001$ & $0.433(0.285-0.659)$ \\
VIII & 134 & 940 & 0.066 & $0.708(0.490-1.023)$ & 0.003 & $0.563(0.385-0.825)$ \\
IX & 121 & 562 & 0.002 & Ref. & $<0.001$ & Ref. \\
X & 83 & 2,100 & 0.005 & $0.490(0.300-0.802)$ & 0.002 & $0.457(0.279-0.749)$ \\
XI & 215 & 1,294 & 0.010 & $0.625(0.438-0.893)$ & 0.001 & $0.541(0.376-0.780)$ \\
XII & 78 & 552 & 0.006 & Ref. & 0.001 & Ref. \\
\hline
\end{tabular}

${ }^{a}$ Adjusted for age and Tumour-Node-Metastasis stage. MST, median survival time; HR, hazard ratio; CI, confidence interval; ADRA1, adrenergic receptor $\alpha 1$; Ref, reference group.
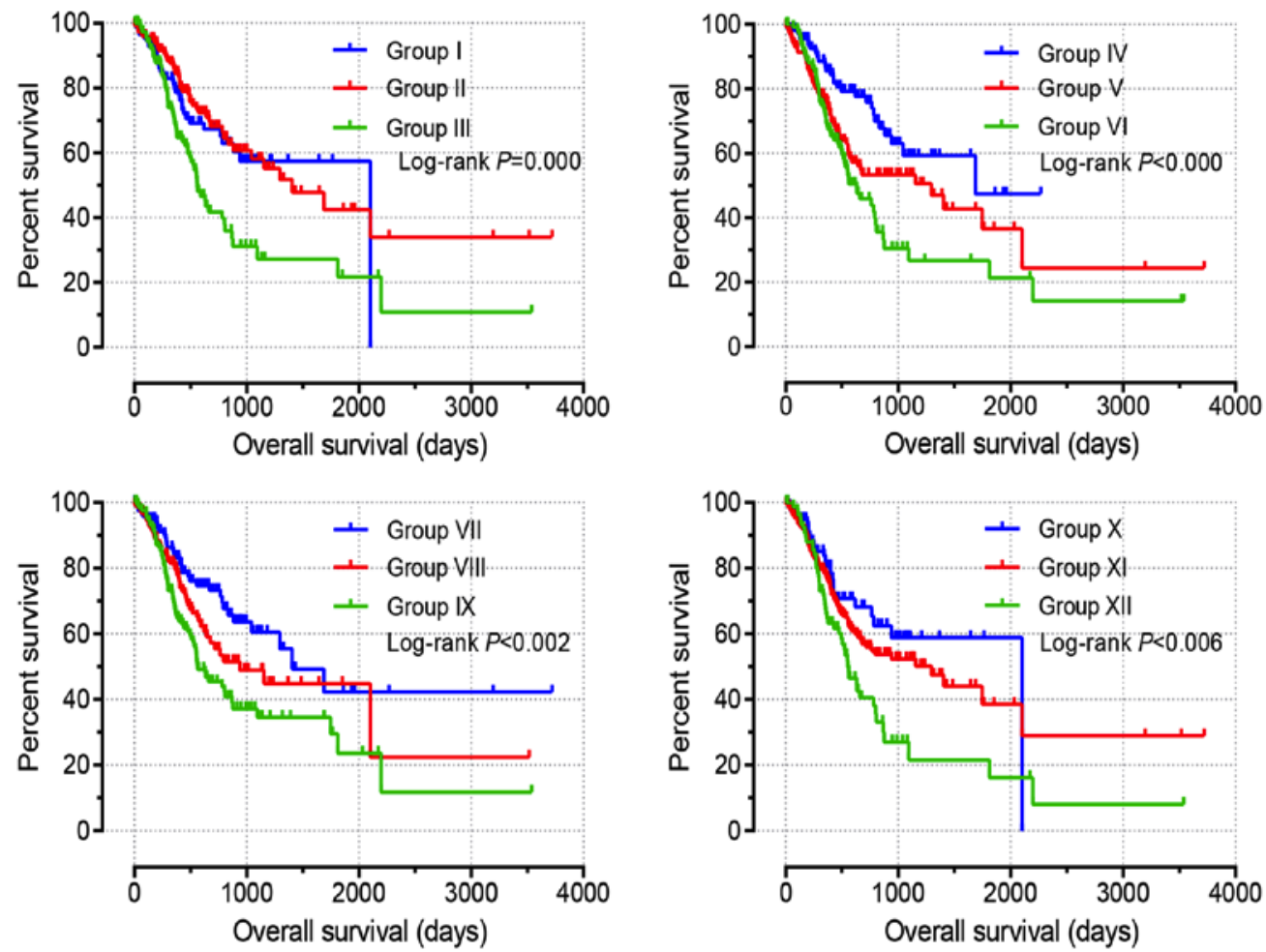

Figure 3. Survival curves for the joint-effects analysis of the combination of ADRA1 subfamily genes expression. Groups are listed in Table I. ADRA1, adrenergic receptor $\alpha 1$.

It is understood that $\mathrm{NE} / \mathrm{E}$ exert their physiological functions predominantly by the $\alpha$ and $\beta$ adrenergic receptors (30). The ability of NE/E to induce invasion and anoikis resistance of cancer cells can be mediated by both ADRA1A and ADRB2 receptors (31). ADRA1 mediates the role of endogenous adrenaline and NE in multiple target cells. NE induces proton release and calcium flux, and activates phospholipase C, PKC and extracellular signal-regulated kinase $1 / 2$ pathways to promote cell proliferation (32). In addition, ADRs transactivate epidermal growth factor receptor (EGFR) signalling via specific matrix metalloproteinases (MMPs) or a disintegrin and metalloproteinase hydrolysis of latent ligands, including heparin-binding EGF-like growth factor (HB-EGF) (33). For the first time, Li et al (30) demonstrated that the ADRA1A and ADRB2 can inhibit EGFR signalling in cancer. EGFR is overexpressed in the majority of adenocarcinoma and 
squamous cell carcinoma cases and can be selectively targeted by pharmacological inhibitors currently used in the clinic (34).

It has been reported that ADRA1 can promote the metastasis of cancer, and continuously activated ADRA induces cell apoptosis via p53 (35). The tumour suppressor protein p53 serves an important role in cellular regulation and acts as an important mediator of apoptotic cell death (36). Evidence suggests that p53 not only induces transcription of pro-apoptotic proteins but also activates the mitochondrial cell death pathway (37). Previous studies have demonstrated that ADRA1A is also associated with reactive oxygen species (ROS) production via the EGFR and the nicotinamide adenine dinucleotide phosphate oxidase signalling pathways (38).

However, little is understood regarding the association between ADRA1 mRNA expression and the prognosis of GC. Using data from TCGA and GEO databases that included mRNA expression profiles of the ADRA1 genes and the associated clinical information from patients with GC, the present study investigated the associations between ADRA1 family members expression and patient prognosis. In addition, the current study assessed whether any ADRA1 genes, alone or in combination, could be used as biomarkers for predicting the prognosis of GC. The results suggested that the expression levels of ADRA1A in normal tissue were higher compared with that in primary tumour tissue. In addition, survival analysis demonstrated that low expression levels of ADRA1A, ADRA1B or ADRA1D were associated with a favourable OS in patients with GC. Furthermore, joint-effects analysis demonstrated that the combination of low levels of all three ADRA1A, ADRA1B and ADRA1D was significantly associated with a favourable OS. By contrast, the combination of high expression levels of ADRA1A, ADRA1B and ADRA1D was associated with a poor OS.

Furthermore, functional analysis and KEGG enrichment identified specific signalling pathways associated with ADRA1 genes including 'Calcium signalling pathway', 'cGMP-PKG signalling pathway' and mitogen-activated protein kinase (MAPK) signalling. These pathways serve important roles in cancer. For example, studies have demonstrated that numerous biological functions are regulated by MAPK signalling, including cell proliferation, apoptosis and metastasis $(39,40)$. In addition, cGMP/PKGI $\beta$ regulates breast cancer cell migration and invasion through the actin/myosin-associated protein, caldesmon (CaD) (41). Analysis of gene-gene interactions showed that certain genes were associated with members of the ADRA1 subfamily [G protein-coupled receptor kinases (GRK)1, GRK4, GRK5, GRK6, GRK7, ARRB1, DRD4, GNAQ, ADRBK2] and some of these genes serve important roles in the regulation of tumour biology. For example, GRKs can modulate GPCR signalling by interacting with the ligand-activated GPCR and phosphorylating its intracellular domain (42). It has been demonstrated that GPCRs affect multiple aspects of cancer biology, such as vascular remodelling, invasion and migration (42).

Peng et al (43) reported that ADRA1A was highly expressed in the peripheral serum of patients with hysterocarcinoma and associated with tumour stage and lymph node metastasis status. Powe et al (44) demonstrated that ADRA1B expression was associated with breast cancer progression and prognosis. Notably, the expression levels of ADRA1A were higher in normal gastric tissues compared with primary gastric tumour tissues in the current study. However, the present study also revealed that low levels of ADRA1 were favourable for patient outcome. There may be a number of reasons for these contradictory results. Firstly, it is understood that certain genes act as tumour suppressors during the early stage of tumorigenesis, while they serve as tumour promoters in later stages. For example, transforming growth factor- $\beta$ (TGF- $\beta$ ) is a cytokine that exhibits dual activities in numerous types of cancer (45). TGF- $\beta$ is an important mediator of cancer invasion, metastasis and angiogenesis; however, it also exhibits antitumor functions (46). Another possible explanation may be that ADRA1 serves different roles in gastric tumour tissue and in normal tissue. Despite the low expression of ADRA1 in gastric cancer tumour tissues, it also serves a role in promoting cancer cell proliferation and metastasis (47). Therefore, the present results require further experimental validation in order to fully elucidate the role of ADRA1 in GC.

In conclusion, the results of the present study demonstrated that the expression levels of ADRA1A, ADRA1B and ADRA1D were associated with the prognosis of gastric cancer. High expression levels of the ADRA1 family were associated with a poor prognosis and a significant reduction in survival rate. These results suggested that an increased expression of the ADRA1 family may somehow promote tumour metastasis. Accordingly, the levels of ADRA1 genes may serve as potential markers for prognosis evaluation.

The present study has certain limitations. Firstly, clinical information and gene expression data used in the study were obtained from a public database and the data were limited. Second, the low expression of ADRA1 in gastric tumour tissues and its role in predicting prognosis is difficult to understand. Due to insufficient funds, the present study did not perform verification experiments. Therefore, the specific mechanism of ADRA1 in gastric cancer tissues requires further experimental validation. Despite these limitations, to the best of our knowledge, the current study is the first to systematically investigate the diagnostic and prognostic values of ADRA1 genes in GC. In summary, ADRA1 genes may serve as potential novel indicators for the prediction of the prognosis of patients with GC.

\section{Acknowledgements}

Not applicable.

\section{Funding}

The present study was supported by the National Natural Science Foundation of China (grant no. 81760521).

\section{Availability of data and materials}

The datasets used and/or analyzed during the present study are available from the author on reasonable request.

\section{Authors' contributions}

JC, TW and MH designed the study, YY and ZL performed the analysis and prepared the manuscript. TW wrote the 
manuscript. WW performed the analysis. YQ and HL helped perform the analysis with constructive discussions. All authors approved the final version of the manuscript.

\section{Ethics approval and consent to participate}

Not applicable.

\section{Patient consent for publication}

Not applicable.

\section{Competing interests}

The authors declare that they have no competing interests.

\section{References}

1. Van Cutsem E, Sagaert X, Topal B, Haustermans K and Prenen H: Gastric cancer. Lancet 388: 2654-2664, 2016.

2. Siegel RL, Miller KD and Jemal A: Cancer statistics, 2015. CA Cancer J Clin 65: 5-29, 2015.

3. Ye J, Xu J, Li Y, Huang Q, Huang J, Wang J, Zhong W, Lin X, Chen W and Lin X: DDAH1 mediates gastric cancer cell invasion and metastasis via Wnt/ $\beta$-catenin signaling pathway. Mol Oncol 11: 1208-1224, 2017.

4. Harris AM, Warner BW, Wilson JM, Becker A, Rowland RG, Conner W, Lane M, Kimbler K, Durbin EB, Baron AT and Kyprianou N: Effect of alpha1-adrenoceptor antagonist exposure on prostate cancer incidence: An observational cohort study. J Urol 178: 2176-2180, 2007.

5. Algazi M, Plu-Bureau G, Flahault A, Dondon MG and Le MG: Is beta-blocker treatment associated with a decrease in the risk of cancer. Lett Drug Des Discov 3: 653-661, 2006.

6. Entschladen F, Drell TL IV, Lang K, Joseph J and Zaenker KS: Tumour-cell migration, invasion, and metastasis: Navigation by neurotransmitters. Lancet Oncol 5: 254-258, 2004.

7. Entschladen F, Drell TL IV, Lang K, Joseph J and Zaenker KS: Neurotransmitters and chemokines regulate tumor cell migration: Potential for a new pharmacological approach to inhibit invasion and metastasis development. Curr Pharm Des 11: 403-411, 2005.

8. Lang K, Drell TL IV, Lindecke A, Niggemann B, Kaltschmidt C, Zaenker KS and Entschladen F: Induction of a metastatogenic tumor cell type by neurotransmitters and its pharmacological inhibition by established drugs. Int J Cancer 112: 231-238, 2004.

9. Dorn GW II and Liggett SB: Pharmacogenomics of beta-adrenergic receptors and their accessory signaling proteins in heart failure. Clin Transl Sci 1: 255-262, 2008.

10. Rosini M, Bolognesi ML, Giardina D, Minarini A, Tumiatti V and Melchiorre C: Recent advances in alpha1-adrenoreceptor antagonists as pharmacological tools and therapeutic agents. Curr Top Med Chem 7: 147-162, 2007.

11. Hein $P$ and Michel MC: Signal transduction and regulation: Are all alpha1-adrenergic receptor subtypes created equal? Biochem Pharmacol 73: 1097-1106, 2007.

12. Docherty JR: Subtypes of functional alpha1-adrenoceptor. Cell Mol Life Sci 67: 405-417, 2010.

13. Hubbard KB and Hepler JR: Cell signalling diversity of the Gqalpha family of heterotrimeric G proteins. Cell Signal 18: 135-150, 2006.

14. Theroux TL, Esbenshade TA, Peavy RD and Minneman KP: Coupling efficiencies of human alpha 1-adrenergic receptor subtypes: Titration of receptor density and responsiveness with inducible and repressible expression vectors. Mol Pharmacol 50: $1376-1387,1996$.

15. Fitzgerald PJ: Is norepinephrine an etiological factor in some types of cancer? Int J Cancer 124: 257-263, 2009.

16. Adissu HA and Schuller HM: Antagonistic growth regulation of cell lines derived from human lung adenocarcinomas of Clara cell and aveolar type II cell lineage: Implications for chemoprevention. Int J Oncol 24: 1467-1472, 2004.
17. Gobbi PG, Villano L, Pozzoli D, Bergonzi M, Vanoli A, Tava F, Dionigi P and Corazza GR: Improving the AJCC/TNM classification for use in early gastric cancer. J Gastrointest Surg 15: 935-941, 2011.

18. In H, Solsky I, Palis B, Langdon-Embry M, Ajani J and Sano T: Validation of the 8th edition of the AJCC TNM staging system for gastric cancer using the national cancer database. Ann Surg Oncol 24: 3683-3691, 2017.

19. Roessler S, Jia HL, Budhu A, Forgues M, Ye QH, Lee JS, Thorgeirsson SS, Sun Z, Tang ZY, Qin LX and Wang XW: A unique metastasis gene signature enables prediction of tumor relapse in early-stage hepatocellular carcinoma patients. Cancer Res 70: 10202-10212, 2010.

20. Roessler S, Long EL, Budhu A, Chen Y, Zhao X, Ji J, Walker R, Jia HL, Ye QH, Qin LX, et al: Integrative genomic identification of genes on $8 \mathrm{p}$ associated with hepatocellular carcinoma progression and patient survival. Gastroenterology 142: 957-966. e12, 2012.

21. Shaul YD, Yuan B, Thiru P, Nutter-Upham A, McCallum S, Lanzkron C, Bell GW and Sabatini DM: MERAV: A tool for comparing gene expression across human tissues and cell types. Nucleic Acids Res 44 (D1): D560-D566, 2016.

22. Anaya J: OncoLnc: Linking TCGA survival data to mRNAs, miRNAs, and lncRNAs. PeerJ Comp Sci 2: e67, 2016.

23. Huang da W, Sherman BT and Lempicki RA: Bioinformatics enrichment tools: Paths toward the comprehensive functional analysis of large gene lists. Nucleic Acids Res 37: 1-13, 2009.

24. Warde-Farley D, Donaldson SL, Comes O, Zuberi K, Badrawi R, Chao P, Franz M, Grouios C, Kazi F, Lopes CT, et al: The GeneMANIA prediction server: Biological network integration for gene prioritization and predicting gene function. Nucleic Acids Res 38: W214-W220, 2010.

25. Mínguez B, Hoshida Y, Villanueva A, Toffanin S, Cabellos L, Thung S, Mandeli J, Sia D, April C, Fan JB, et al: Gene-expression signature of vascular invasion in hepatocellular carcinoma. J Hepatol 55: 1325-1331, 2011.

26. Magnon C, Hall SJ, Lin J, Xue X, Gerber L, Freedland SJ and Frenette PS: Autonomic nerve development contributes to prostate cancer progression. Science 341: 1236361, 2013.

27. Schuller HM: Neurotransmitter receptor-mediated signaling pathways as modulators of carcinogenesis. Prog Exp Tumor Res 39: 45-63, 2007.

28. Thaker PH, Han LY, Kamat AA, Arevalo JM, Takahashi R, Lu C, Jennings NB, Armaiz-Pena G, Bankson JA, Ravoori M, et al: Chronic stress promotes tumor growth and angiogenesis in a mouse model of ovarian carcinoma. Nat Med 12: 939-944, 2006.

29. Wan D, Gong Y, Qin W, Zhang P, Li J, Wei L, Zhou X, Li H, Qiu X, Zhong F, et al: Large-scale cDNA transfection screening for genes related to cancer development and progression. Proc Natl Acad Sci USA 101: 15724-15729, 2004.

30. Li J, Yang XM, Wang YH, Feng MX, Liu XJ,Zhang YL, Huang S, Wu Z, Xue F, Qin WX, et al: Monoamine oxidase A suppresses hepatocellular carcinoma metastasis by inhibiting the adrenergic system and its transactivation of EGFR signaling. J Hepatol 60: 1225-1234, 2014

31. Perez DM (ed): The adrenergic receptors: In the 21st century. NewYork, Humana Press, 2006.

32. Liu W, Wang X, Mei Z, Gong J, Huang L, Gao X, Zhao Y, Ma J and Qian L: BNIP3L promotes cardiac fibrosis in cardiac fibroblasts through $\left[\mathrm{Ca}^{2+}\right] \mathrm{i}-\mathrm{TGF}-\beta-\mathrm{Smad} 2 / 3$ pathway. Sci Rep 7: 1906, 2017.

33. Oganesian A, Yarov-Yarovoy V, Parks WC and Schwinn DA: Constitutive coupling of a naturally occurring human alpha1aadrenergic receptor genetic variant to EGFR transactivation pathway. Proc Natl Acad Sci USA 108: 19796-19801, 2011.

34. Salazar R and Ciardiello F: Optimizing anti-EGFR therapy in colorectal cancer. Clin Cancer Res 21: 5415-5416, 2015.

35. García-Cazarín ML, Smith JL, Clair DK and Piascik MT: The alpha1D-adrenergic receptor induces vascular smooth muscle apoptosis via a p53-dependent mechanism. Mol Pharmacol 74: 1000-1007, 2008.

36. Okada H and Mak TW: Pathways of apoptotic and non-apoptotic death in tumour cells. Nat Rev Cancer 4: 592-603, 2004.

37. Liu B, Chen Y and St Clair DK: Ros and p53: A versatile partnership. Free Radic Biol Med 44: 1529-1535, 2008.

38. XiaoL, PimentelDR, Wang J, Singh K, Colucci WS and Sawyer DB: Role of reactive oxygen species and NAD $(\mathrm{P}) \mathrm{H}$ oxidase in alpha(1)-adrenoceptor signaling in adult rat cardiac myocytes. Am J Physiol Cell Physiol 282: C926-C934, 2002. 
39. Yoda Y, Takeshima H, Niwa T, Kim JG, Ando T, Kushima R, Sugiyama T, Katai H, Noshiro $\mathrm{H}$ and Ushijima T: Integrated analysis of cancer-related pathways affected by genetic and epigenetic alterations in gastric cancer. Gastric Cancer 18: 65-76, 2015.

40. Wang Z, Wang W, Xu S, Wang S, Tu Y, Xiong Y, Mei J and Wang C: The role of MAPK signaling pathway in the Her-2-positive meningiomas. Oncol Rep 36: 685-695, 2016.

41. Schwappacher R, Rangaswami H, Su-You J, Hassad A, Spitler R and Casteel DE: cGMP-dependent protein kinase $\mathrm{I} \beta$ regulates breast cancer cell migration and invasion via interaction with the actin/myosin-associated protein caldesmon. J Cell Sci 126: 1626-1636, 2013

42. Yu S, Sun L, Jiao Y and Lee LTO: The role of G protein-coupled receptor kinases in cancer. Int J Biol Sci 14: 189-203, 2018.

43. Peng L, Peng W, Hu P and Zhang HF: Clinical significance of expression levels of serum ADRA1A in hysterocarcinoma patients. Oncol Lett 15: 9162-9166, 2018.
44. Powe DG, Voss MJ, Habashy HO, Zänker KS, Green AR, Ellis IO and Entschladen F: Alpha- and beta-adrenergic receptor (AR) protein expression is associated with poor clinical outcome in breast cancer: An immunohistochemical study. Breast Cancer Res Treat 130: 457-463, 2011.

45. Huang JJ and Blobe GC: Dichotomous roles of TGF- $\beta$ in human cancer. Biochem Soc Trans 44: 1441-1454, 2016.

46. Costanza B, Umelo IA, Bellier J, Castronovo V and Turtoi A: Stromal modulators of TGF- $\beta$ in cancer. J Clin Med 6: pii: E7, 2017.

47. Tatsuta M, Iishi H, Baba M, Yano H, Sakai N, Uehara H, Hirasawa R and Nakaizumi A: Alpha1-adrenoceptor stimulation enhances experimental gastric carcinogenesis induced by N-methyl-N'-nitro-N-nitrosoguanidine in Wistar rats. Int J Cancer 77: 467-469, 1998.

(i) (9) This work is licensed under a Creative Commons Attribution-NonCommercial-NoDerivatives 4.0 International (CC BY-NC-ND 4.0) License. 